\title{
Arritmias ventriculares como manifestación de sarcoidosis cardiaca primaria
}

\author{
Jhan Altamar ${ }^{\mathrm{a}, \mathrm{b}, *}$, Carlos Tapias $^{\mathrm{a}, \mathrm{b}}$, Diego Rodríguez $^{\mathrm{a}, \mathrm{b}}$ y Luis Sáenz ${ }^{\mathrm{a}, \mathrm{b}}$ \\ a Centro internacional de arritmias "Andrea Natale", Fundación Cardioinfantil-Instituto de Cardiología, Bogotá, Colombia \\ b Universidad de La Sabana, Bogotá, Colombia
}

Recibido el 15 de julio de 2016; aceptado el 6 de febrero de 2017

Disponible en Internet el 16 de junio de 2017

\section{PALABRAS CLAVE \\ Miocarditis; \\ Taquicardia; \\ Bloqueo cardíaco; \\ Bloqueo de rama}

\section{KEYWORDS}

Myocarditis;

Tachycardia;

Heart block;

Branch block

\begin{abstract}
Resumen La afectación cardíaca en pacientes con sarcoidosis está siendo cada vez más reconocida y se asocia con mal pronóstico. Aunque en su patogénesis están implicados los factores ambientales y genéticos, la etiología de la sarcoidosis cardíaca no es clara. Las manifestaciones clínicas incluyen alteraciones de la conducción aurículo-ventricular, arritmias e insuficiencia cardíaca congestiva. Es una entidad extremadamente difícil de diagnosticar debido a que las manifestaciones clínicas son inespecíficas, y la sensibilidad y la especificidad de las modalidades de diagnóstico son limitadas. El tratamiento cardíaco óptimo no ha sido bien definido, y aunque los corticoides siguen siendo el pilar del manejo, hay poca evidencia de la dosis o la duración de la terapia. Se expone el caso de una paciente con sarcoidosis cardíaca aislada, que debutó con extrasístoles ventriculares y progresó a falla cardiaca y arritmias ventriculares sostenidas. (c) 2017 Sociedad Colombiana de Cardiología y Cirugía Cardiovascular. Publicado por Elsevier España, S.L.U. Este es un artículo Open Access bajo la licencia CC BY-NC-ND (http:// creativecommons.org/licenses/by-nc-nd/4.0/).
\end{abstract}

\section{Ventricular arrhythmias as a first sign of primary cardiac sarcoidosis}

Abstract Cardiac involvement is increasingly being observed in patients with sarcoidosis and is associated with a poor prognosis. Although environmental and genetic factors play a part in its pathogenesis, the aetiology of cardiac sarcoidosis is still not clear. The clinical signs include anomalies in atrial-ventricular conduction, arrhythmias, and congestive cardiac failure. It is an extremely difficult condition to diagnose as the clinical signs are vague, and the sensitivity and specificity of the diagnostic models are limited. There is no well-defined optimum cardiac treatment, and although corticosteroids continue to be the mainstay of its management, there

\footnotetext{
* Autor para correspondencia.

Correo electrónico: jcarlomed@gmail.com (J. Altamar).
} 
is little evidence on the dose or duration of the treatment. It is presented a case of a patient with isolated cardiac sarcoidosis that debuted with ventricular extrasystoles and progressed to cardiac failure and sustained ventricular arrhythmias.

(C) 2017 Sociedad Colombiana de Cardiología y Cirugía Cardiovascular. Published by Elsevier España, S.L.U. This is an open access article under the CC BY-NC-ND license (http:// creativecommons.org/licenses/by-nc-nd/4.0/).

\section{Caso}

Mujer de 62 años, con episodio de dolor torácico acompañado de palpitaciones precordiales asociado a inestabilidad hemodinámica, sin registro electrocardiográfico del centro de referencia. Fue remitida a la institución por cuadro de choque de posible etiología cardiogénica a causa de infarto agudo de miocardio.

Cinco años atrás, a raíz de extrasístoles ventriculares (fig. 1) sintomáticas, se había realizado monitorización Holter en la que se evidenciaba carga arrítmica del 13\%, sin respuesta al manejo con betabloqueadores.

A su ingreso se ordenaron paraclínicos, incluida troponina ultrasensible cuyo resultado fue positivo, electrocardiograma en ritmo sinusal con necrosis en cara lateral alta, ecocardiograma transtorácico que mostró dilatación moderada con hipocinesias de la pared lateral e inferior del ventrículo izquierdo y fracción de eyección del 35\%. Se hizo coronariografía en la que se evidenciaron arterias epicárdicas sanas. Durante su hospitalización se reprodujeron los síntomas que motivaron su consulta, acompañados de inestabilidad hemodinámica. En nuevo electrocardiograma (fig. 2) se registró taquicardia ventricular sostenida monomórfica.

Dado que no existía una causa bien establecida de la cardiopatía dilatada se decidió ampliar el estudio diagnóstico con resonancia magnética cardíaca gatillada, la cual mostró depósito focal de gadolinio transmural en el septum basal, a nivel subepicárdico en los segmentos basal lateral, anterior y apical inferolateral. Se tomó biopsia endomiocárdica (fig. 3), mediante la cual se confirmó el diagnóstico de sarcoidosis, descartándose compromiso extracardíaco, y se inició tratamiento con corticoides. Aunque no se realizó estudio con tomografía con emisión de positrones, se consideró que los hallazgos histológicos sugerían inflamación activa, hecho que justificaba el inicio de dicha terapia. Se indicó egreso hospitalario previo implante de cardiodesfibrilador para prevención secundaria de muerte súbita.

\section{Discusión}

Gran parte de los pacientes con compromiso cardíaco por sarcoidosis pasa inadvertida, pues solo en el $5 \%$ de los casos se hace evidente en cuanto a la clínica. Adicionalmente, en $25 \%$ de las autopsias de pacientes con sarcoidosis, se documentan granulomas cardiacos ${ }^{1}$.

Las manifestaciones cardíacas varían ampliamente de paciente a paciente; sin embargo hasta un $65 \%$ del compromiso está representado por alteraciones del sistema de conducción, dentro de las que se incluyen bloqueo aurículo-ventricular, cambio de la conducción intraventricular y muerte súbita ${ }^{1}$.

Ante la sospecha de sarcoidosis cardíaca, la recomendación emitida en el consenso de expertos de la Sociedad del ritmo cardíaco (HRS), es realizar un examen físico completo, electrocardiograma de 12 derivaciones y ecocardiograma ${ }^{2}$. La búsqueda se lleva a cabo en pacientes con compromiso extracardíaco confirmado desde el punto de vista histopatológico o ante manifestaciones cardíacas específicas, dentro de las que se incluyen bloqueo aurículo-ventricular de segundo grado Mobitz II y bloqueo aurículo-ventricular completo en pacientes menores de 60 años sin una causa clara que explique dichas alteraciones, además de taquicardia ventricular sostenida $0 \mathrm{no}^{2}$. La paciente del caso debutó con extrasístoles ventriculares con morfología que sugiere origen en el tracto de salida del ventrículo derecho, por lo que inicialmente no se sospechó sarcoidosis cardíaca; podría tratarse de un hallazgo coincidencial o como manifestación inicial de la enfermedad con progresión de la misma. Teniendo en cuenta la poca especificidad de las manifestaciones cardíacas y las dificultades que esto representa para el diagnóstico de esta entidad, es vital tener en mente la variedad de alteraciones en la que se manifiesta y hacer seguimientos periódicos en los casos dudosos. Aunque es claro que la biopsia endomiocárdica es la prueba de oro en el diagnóstico de la sarcoidosis cardíaca, la resonancia magnética cardíaca gatillada y la tomografía por emisión de positrones con deoxifluroglucosa, se convierten en herramientas fundamentales en la estrategia de estudio y en el caso de esta última, en pieza clave para indicar el uso de esteroides y para evaluar la respuesta al manejo ${ }^{2}$.

El elemento fundamental en la inmunopatogénisis es la activación de la respuesta inmune celular y humoral, en cuyo caso los linfocitos T CD4 hacen parte del andamiaje de esta respuesta, con la formación de granulomas no caseificantes y la fibrosis tisular acompañante ${ }^{3}$. Los corticoides pueden detener la progresión de la enfermedad cardíaca y mejorar la supervivencia por ralentización de la progresión de la inflamación y la fibrosis ${ }^{4}$; podrían lograrse resultados superiores si se inician temprano en el curso de la enfermedad ${ }^{5,6}$. Son más beneficiosos en pacientes con bloqueos cardíacos tercer grado Mobitz II o ectopia ventricular frecuente, taquicardia ventricular no sostenida o arritmias ventriculares sostenidas e inflamación del miocardio ${ }^{7,8}$. El consenso de expertos sobre arritmias asociadas a sarcoidosis cardíaca recomienda el manejo con esteroides en pacientes con bloqueo aurículo-ventricular Mobitz ॥ o bloqueo cardíaco de 


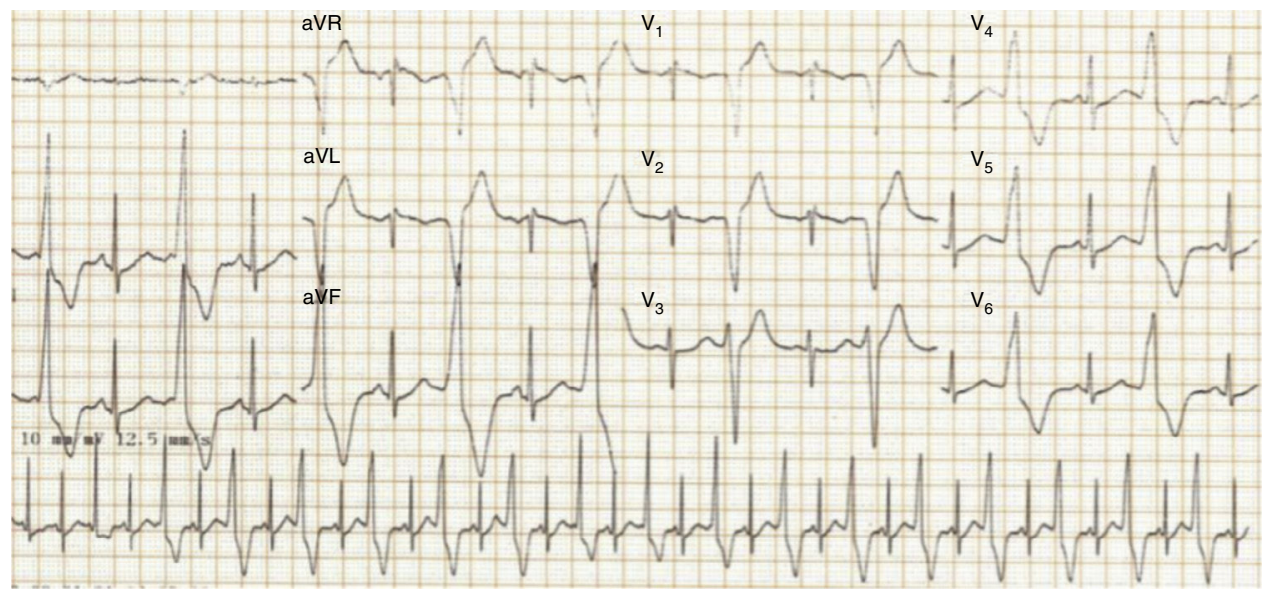

Figura 1 Electrocardiograma ritmo sinusal, extrasístoles ventriculares monomórficas con patrón de bloqueo de rama izquierda con transición tardía y eje inferior derecho.

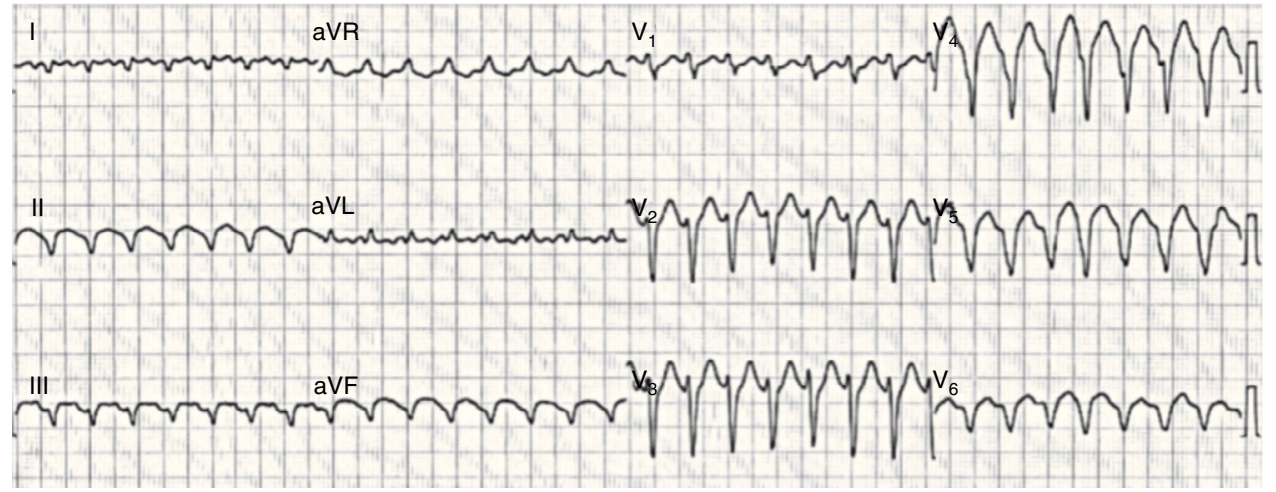

Figura 2 Taquicardia ventricular monomórfica; sugiere origen en la pared inferolateral hacia el aspecto apical del ventrículo izquierdo.

tercer grado ${ }^{2}$. En cuanto a las arritmias ventriculares, en el mismo documento se recomienda el inicio de la terapia inmunosupresora en el escenario de extrasístoles ventriculares frecuentes, taquicardia ventricular no sostenida y arritmias ventriculares sostenidas, para lo cual se debe evaluar inflamación miocárdica con tomografía con emisión de positrones con deoxifluroglucosa, como condición previa a la implementación de esta intervención ${ }^{2}$.

Dentro de las recomendaciones para el manejo de las alteraciones de la conducción, el documento emitido por los expertos indica la implantación de un dispositivo de estimulación incluso si el bloqueo aurículo-ventricular se

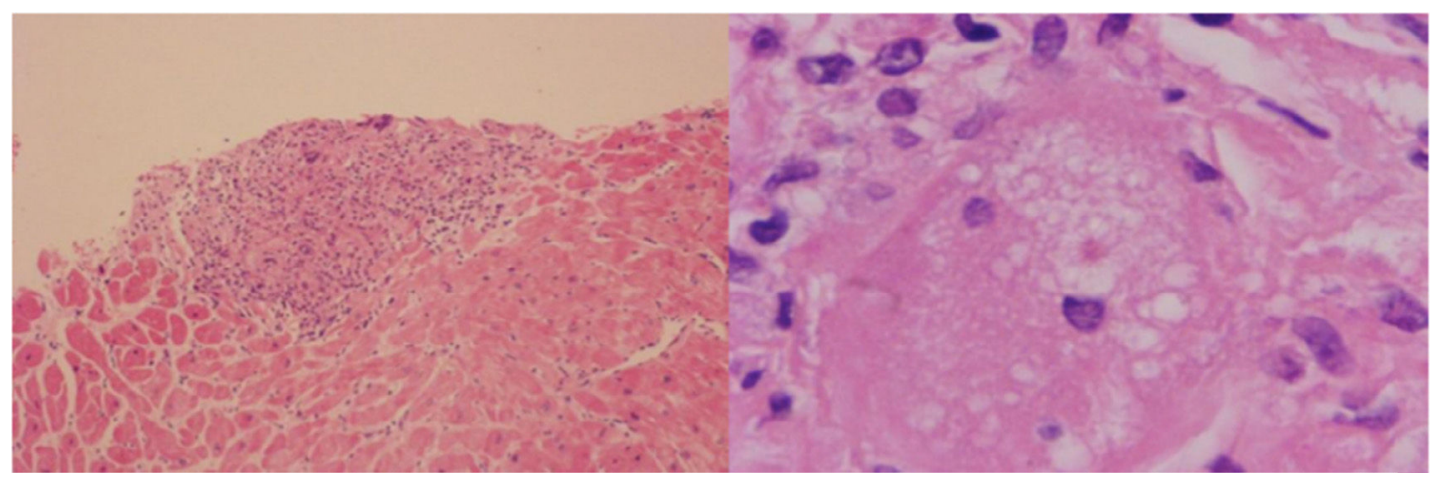

Figura 3 Tejido biopsia cardíaca. Se observan fragmentos de miocardio con compromiso por múltiples gránulos epiteliales no necrotizantes focales y coalescentes, que involucran aproximadamente el $30 \%$ del tejido analizado. Los gránulos contienen numerosas células gigantes multinucleadas y en las áreas confluentes se asocian con fibrosis intersticial. Las tinciones fueron negativas para micobacterias y hongos. 
revierte en forma transitoria, en pacientes con bloqueo aurículo-ventricular Mobitz $\|$ y bloqueo de tercer grado ${ }^{2}$. Se recomienda, además, el implante de un cardiodesfibrilador en pacientes con indicación de implante de dispositivo de estimulación ${ }^{2}$. Al respecto emite dos recomendaciones clase I: la presencia de arritmias ventriculares sostenidas, incluido paro cardíaco previo y pacientes con fracción de eyección menor o igual a $35 \%$ a pesar de terapia médica óptima y un periodo de inmunosupresión (en caso de documentarse inflamación). Este periodo de tiempo debe individualizarse y ser al menos de tres meses ${ }^{2}$.

Hay un grupo de pacientes que se ubica en zona gris, con fracción de eyección mayor al 35\%, sin indicación de implante de cardiodesfibrilador en el abordaje inicial, quienes pueden ser estratificados mediante evaluación de la función sistólica, resonancia magnética cardíaca o estudio electrofisiológico, con el fin de definir la necesidad de implante del mismo ${ }^{2}$.

\section{Conclusión}

La sarcoidosis es una enfermedad sistémica, que con frecuencia acarrea compromiso cardíaco, en cuyo caso las arritmias son las manifestaciones más comunes, pudiéndose presentar en el contexto de compromiso multisistémico y raras veces como sarcoidosis cardíaca aislada. Tener en mente las formas de presentación del compromiso cardíaco, al igual que efectuar un abordaje diagnóstico y terapéutico, agresivo y oportuno podría impactar el curso y pronóstico de esta entidad. El manejo de bradiarritmias y taquiarritmias ventriculares incluye el uso de esteroides en conjunto con dispositivos de estimulación cardíaca y cardiodesfibrilador.

\section{Responsabilidades éticas}

Protección de personas y animales. Los autores declaran que para esta investigación no se han realizado experimentos en seres humanos ni en animales.
Confidencialidad de los datos. Los autores declaran que han seguido los protocolos de su centro de trabajo sobre la publicación de datos de pacientes.

Derecho a la privacidad y consentimiento informado. Los autores declaran que en este artículo no aparecen datos de pacientes.

\section{Conflictos de intereses}

Ninguno.

\section{Bibliografía}

1. Houston B, Mukherjee M. Cardiac sarcoidosis: clinical manifestations, imaging characteristics, and therapeutic approach. Clin Med INSIGHTS Cardiol. 2014;8:31-7.

2. Birnie DH, Sauer WH, Bogun F, Cooper JM, Culver DA, Duvernoy $\mathrm{CS}$, et al. HRS expert consensus statement on the diagnosis and management of arrhythmias associated with cardiac sarcoidosis. Hear Rhythm. 2014;11:1305-24.

3. Iannuzzi M, Rybicki B, Teirstein A. Sarcoidosis N Engl J Med. 2007;913:908-13.

4. Doughan AR, Williams BR. Cardiac sarcoidosis. Heart. 2006;92:282-8.

5. Kim JS, Judson MA, Donnino R, Gold M, Cooper LT, Prystowsky EN, et al. Cardiac sarcoidosis. Am Heart J. 2009;157:9-21.

6. Yazaki Y, Isobe M, Hiroe M, Morimoto S, Hiramitsu S, Nakano T, et al. Prognostic determinants of long-term survival in japanese patients with cardiac sarcoidosis treated with prednisone. Am J Cardiol. 2001;88:1006-10.

7. Sadek MM, Yung D, Birnie DH, Beanlands RS, Nery PB. Corticosteroid therapy for cardiac sarcoidosis: a systematic review. Can J Cardiol [Internet]. Canadian Cardiovascular Society. 2013;29:1034-41.

8. Yodogawa K, Seino Y, Ohara T, Takayama H, Katoh T, Mizuno K. Effect of corticosteroid therapy on ventricular arrhythmias in patients with cardiac sarcoidosis. Ann Noninvasive Electrocardiol. 2011;16:140-7. 